\title{
Evaluation of epicardial fat tissue thickness in patients with multiple sclerosis
}

\author{
Mehmet Cosgun $^{1} \cdot$ Isa Sincer $^{\mathbf{1}} \cdot$ Yilmaz Gunes $^{1} \cdot$ Zafer Kok $^{\mathbf{1}} \cdot$ Sule Aydın Turkoglu $^{\mathbf{2}}$ \\ ${ }^{I}$ Department of Cardiology, Bolu Abant Izzet Baysal University, Faculty of Medicine, Bolu, Turkey \\ ${ }^{2}$ Department of Neurology, Bolu Abant Izzet Baysal University, Faculty of Medicine, Bolu, Turkey
}

\section{ABSTRACT}

\begin{abstract}
Aim: Multiple sclerosis (MS), which is inflammatory in its pathogenesis, damages the myelin sheath in the central nervous system (CNS) and causes axonal loss. Epicardial fat tissue (EFT), located between the myocardium and the visceral layer of the pericardium, surrounds the heart and several inflammatory cytokines is secreted from this tissue. In this study, we aimed to investigate EFT thickness in MS patients and compared with that of volunteer non-MS subjects.

Methods: A total of 154 subjects comprising 61 MS patients and 93 volunteers matched for gender and age were included in our study. Epicardial fat tissue thickness was measured by echocardiography. All values were compared between groups.

Results: Echocardiographic parameters were similar in both groups. However, the mean EFT thickness was significantly higher in the MS group than in the control group $(p<0.001)$. Epicardial fat tissue thickness was also significantly correlated with the presence of MS $(r=0.33, p<0.001)$.

Conclusion: The results of our study suggest that the increase in epicardial adipose tissue thickness in MS patients may be a predictive factor for cardiovascular disease. However, the clinical significance of this finding and its relevance to MS pathogenesis should be investigated in further studies.
\end{abstract}

Keywords: Multiple sclerosis, epicardial fat tissue, cardiovascular diseases, inflammation, biomarkers.

Dr. Mehmet Cosgun

Department of Cardiology, Bolu Abant Izzet Baysal

University, Faculty of Medicine, Bolu, Turkey

E-mail: coskun44@gmail.com

Received: 2020-11-02 Revised: 2020-11-30

Accepted: 2020-12-12 / Published online: 2021-01-01

\section{Introduction}

Multiple sclerosis (MS), which is inflammatory in its pathogenesis, damages the myelin sheath in the central nervous system (CNS) and causes axonal loss [1,2]. Although its etiology is unknown, genetic and environmental factors are presumed to trigger an autoimmune response, resulting in damage to myelin and axons [3]. However, inflammation might also be neuroprotective or contribute to the repair of damaged tissue within the CNS [4].

Epicardial fat tissue (EFT), located between the myocardium and pericardial visceral layer, surrounds the heart. Embryologically, EFT and abdominal adipose tissue are of the same origin [5]. The levels of lipolysis and lipogenesis in EFT are quite high compared to those of other adipose tissues [6]. Moreover, EFT is in contact with coronary arteries and secretes many inflammatory cytokines [5,7]. Studies have shown that EFT may be an independent risk factor for the presence and severity of cardiovascular disease (CVD), probably through inflammation or vasoactive pathways [8]. 
Inflammatory cytokines play a crucial role in the pathogenesis of MS [9]. MS, which is an autoimmune disease, progresses with demyelination in the CNS [10]. EFT thickness is associated with various autoimmune diseases $[11,12]$, and there are various studies showing the release of various cytokines from EFT [13]. Hence, EFT thickness may be considered a significant parameter in MS patients.

In this study, we aimed to investigate echocardiographic EFT thickness, which may be an indicator of coronary artery disease, in MS patients and compared it with that of normal subjects.

\section{Materials and Methods}

The patients who were admitted to a university hospital between February 2019 and March 2020 were included in the study. The study was approved by Bolu Abant Izzet Baysal local ethics committee (Date: 30/01/2019; Decision number: 2019/27). Written and verbal consent were obtained from all patients.

A total of 154 subjects comprising $61 \mathrm{MS}$ patients and 93 volunteers matched for gender and age were included in our study. The demographic characteristics of the participants were recorded. The laboratory parameters of the patients at the time of admission were recorded from the laboratory database. Echocardiography (echo) was performed on all participants.

\section{Exclusion criteria}

Patients who had a history of coronary, carotid, or peripheral artery atherosclerosis, valvular heart disease, heart failure, arrhythmias or conduction disorders, prosthetic heart valves, pacemaker implantation, significant renal failure, severe liver dysfunction, thyroid disorders, electrolyte imbalances, systemic inflammatory or infectious disease, and poor ultrasound image quality were excluded.

\section{Measurement of epicardial fat thickness}

EFT thickness was measured with a 4-Mhz Vivid S6 transducer (GE Vingmed, N-3191, Horten, Norway). A blinded cardiologist performed the echo under continuous electrocardiographic monitoring in the left lateral position. Measurements were recorded as the average of the values obtained over three cardiac cycles. EFT thickness was noted as an echo-free or hyperechoic area between the epicardium and the visceral layer of the pericardium and reflects visceral adipose tissue [14]. The assessment of EFT thickness was performed from the parasternal long- and shortaxis views of the free wall of the right ventricle. The measurement was made at the level of the aortic annulus perpendicular to the free wall of the right ventricle. The measurements of maximum values were made at the end of diastole. The intraobserver variation for EFT was $<5 \%$.

\section{Statistical analysis}

For all statistical analyses, the 18.0 version of SPSS (Statistical Package for the Social Sciences) for Windows, (Chicago, Illinois, USA) was used. The numerical data were expressed as mean \pm standard deviation.

The difference between the study and the control groups was evaluated by Student t-test for data which were normally distributed. For the data which were not normally distributed Mann-Whitney's U-test was used. The correlation between EFT and MS was evaluated using Spearman's correlation analysis. A pvalue $<0.05$ was considered as significant.

\section{Results}

The clinical risk factors and baseline characteristics of the patients are shown in Table 1. No significant difference was found between the groups in terms of baseline characteristics (Table 1). 
Likewise, echocardiographic findings were similar in both MS and non-MS groups. However, the mean EFT thickness value was found significantly higher in MS group than in non-MS group $(p<0.001) \quad($ Table 2$)$. No significant difference was found between the groups in terms of laboratory parameters such as creatinine, triglyceride, and cholesterol values. The Spearman's correlation test revealed that EFT thickness $(\mathrm{r}=0.33, \mathrm{p}<0.001)$ was significantly correlated with the presence of MS (Table 2, Figure 1).

Table 1. Baseline characteristics of the study groups.

\begin{tabular}{|l|c|c|c|}
\hline Baseline characteristics & Control Groups (n=93) & MS Groups (n=61) & P Value \\
\hline Age (years) & $33 \pm 12$ & $36 \pm 12$ & 0.25 \\
\hline Sex (Male/female), $\mathrm{n}$ & $46 / 47$ & $22 / 39$ & 0.08 \\
\hline SBP (mmHg) & $116 \pm 14$ & $111 \pm 12$ & 0.05 \\
\hline DBP (mmHg) & $71 \pm 9$ & $70 \pm 7$ & 0.64 \\
\hline Body mass index $\left(\mathrm{kg} / \mathrm{m}^{2}\right)$ & $25 \pm 4$ & $25 \pm 3$ & 0.17 \\
\hline Smoking & $19(20 \%)$ & $17(28 \%)$ & 0.27 \\
\hline Diabetes Mellitus & $1(1 \%)$ & $4(5 \%)$ & 0.14 \\
\hline Hypertension & $2(2 \%)$ & 0.16 \\
\hline MS: Multiple sclerosis, DBP: Diastolic Blood Pressure, SD: Standard Deviation, SBP: Systolic Blood Pressure. \\
\hline
\end{tabular}

Table 2. Echocardiographic and laboratory parameters of the study groups.

\begin{tabular}{|c|c|c|c|}
\hline Variables & Control Group (n=93) & MS Group (n=61) & $P$ Value \\
\hline & \multicolumn{2}{|c|}{ Mean \pm SD } & \\
\hline $\mathrm{EF}$ & $66 \pm 3$ & $65 \pm 3$ & 0.36 \\
\hline LVDD $(\mathrm{cm})$ & $4.6 \pm 0.4$ & $4.5 \pm 0.3$ & 0.28 \\
\hline $\operatorname{LVSD}(\mathrm{cm})$ & $2.8 \pm 0.4$ & $2.8 \pm 0.3$ & 0.26 \\
\hline $\mathrm{PW}(\mathrm{cm})$ & $0.86 \pm 12$ & $0.88 \pm 0.12$ & 0.33 \\
\hline IVST(cm) & $0.88 \pm 0.14$ & $0.93 \pm 0.14$ & 0.04 \\
\hline Left atrium & $2.9 \pm 0.5$ & $3.0 \pm 0.4$ & 0.69 \\
\hline Creatinine $(\mathrm{mg} / \mathrm{dl})$ & $0.78 \pm 12$ & $0.78 \pm 11$ & 0.98 \\
\hline Triglyceride $(\mathrm{mg} / \mathrm{dl})$ & $107 \pm 52$ & $107 \pm 59$ & 0.97 \\
\hline \multirow[t]{2}{*}{$\mathrm{EFT}(\mathrm{cm})$} & $0.41 \pm 0.17$ & $0.50 \pm 0.12$ & $<0.001$ \\
\hline & \multicolumn{2}{|c|}{ Median (IQR) } & \\
\hline HDL-cholesterol (mg/dl) & $46(8.8)$ & $51(17.1)$ & 0.07 \\
\hline LDL-cholesterol (mg/dl) & $101(13.7)$ & $102(38.1)$ & 0.79 \\
\hline Total cholesterol (mg/dl) & $169(21)$ & $178(34)$ & 0.08 \\
\hline
\end{tabular}




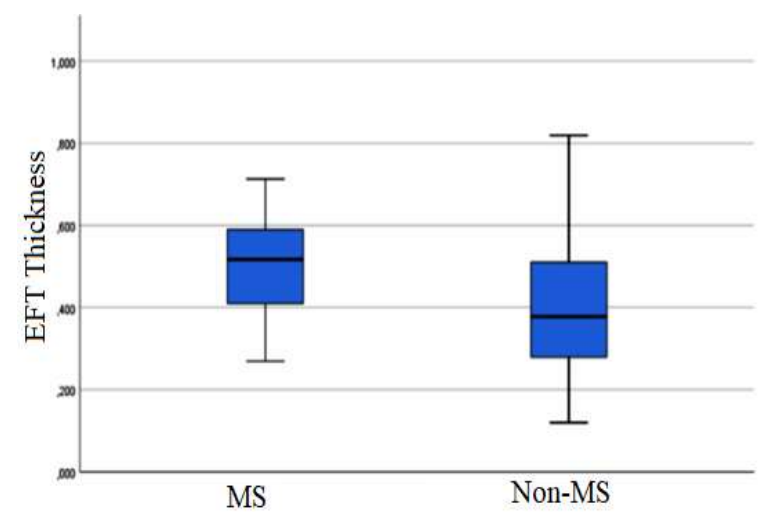

Figure 1. EFT thickness is significantly higher MS group compared to non-MS group.

\section{Discussion}

In the present study it is found that EFT thickness was significantly higher in the MS group than in the non-MS group, and EFT thickness was significantly correlated with MS. There is no consensus on whether MS patients are at greater risk of CVD when compared to the general population [15-18]. While Roshanisefat et al. found no difference between MS and non-MS patients in terms of CVD incidence, Moccia et al. reported that the Framingham General Cardiovascular Disease Risk Score (FR) was associated with the severity, disability, and clinical course of MS [19-20]. In some studies, it has been reported that indirect markers of CVD, such as highsensitive C-reactive protein and carotid intima media thickness (CIMT), were related to MS [21]. Hence, the overall data suggest an increased CVD risk in patients with MS [22].

In the context of the reported studies, it is not clearly understood whether the increase in CVD is related to the presence of obesity, hypertension, hyperlipidemia, or diabetes mellitus in MS. Further studies are needed to solve this issue [22]. An association between EFT thickness, CVD, and metabolic syndrome has been observed in several studies [8,23]. Therefore, our finding of increased EFT thickness in MS patients may provide indirect evidence and a pathway for the association of MS with CVD.

In the early phase of relapsing-remitting MS, demyelination by inflammation predominates, while in the progressive phase, neuroaxonal damage by neurodegeneration predominates [24]. Frischer et al. reported a significant association between inflammation and axonal injury in MS. In older MS patients with longer disease duration, inflammatory infiltrates declined to levels similar to those found in agematched controls [25]. Therefore, although it is not known whether the role of inflammation is causative or protective, there is a strong association between neurodegeneration and inflammation in MS.

Epicardial fat tissue is a paracrine and endocrine tissue and is a source of adiponectin, adrenomedullin, anti-inflammatory adipokines, and several proinflammatory cytokines, such as tumor necrosis factor alpha (TNF- $\alpha$ ), interleukin (IL)-1, IL-6, and nerve growth factor (NGF) [13]. Mazurek et al. [7] examined epicardial and subcutaneous adipose tissues among elective coronary artery bypass grafting surgery patients. Significantly higher mRNA and protein levels of IL-1, IL-6, MCP-1, and TNF- $\alpha$ were detected in EFT when compared to subcutaneous adipose tissue. Coincidentally, IL-6, IL-1, and TNF- $\alpha$ levels are associated with MS, and these cytokines have been found to be related to axonal injury [26]. Additionally, Sharief et al. reported that TNF- $\alpha$ levels are correlated with MS disease progression and disability level [27].

The clinical significance of the inflammatory potential of EFT and its role in other diseases is unclear. Our finding of increased EFT thickness among MS patients may provide new insight into the association between inflammation and 
MS. Nevertheless, future studies are needed to further explore this aspect.

There are some limitations not studied. This is a single-center study with a small sample size, which predisposes it to an inherent selection bias. The accuracy and reproducibility of EFT thickness measurements with MRI and CT are higher than that of echo. Therefore, the use of echo for the measurements of EFT thickness may reduce the reliability of our results. Nonetheless, echo was performed as it is simple and inexpensive. Lastly, since EFT has a threedimensional distribution, echo, which produces a two-dimensional image, may not fully evaluate the total amount of epicardial adipose tissue.

\section{Conclusions}

The results of our study suggest that the increase in epicardial adipose tissue thickness in MS patients may be a predictive factor for cardiovascular disease. To our knowledge, this is the first report about epicardial adipose tissue in MS patients. The clinical significance of this finding and its relevance to MS pathogenesis should be explored in further studies.

Funding: There is no financial support and sponsorship

Conflict of Interest: The authors declare that they have no conflict of interest.

Ethical statement: The study was approved by Bolu Abant Izzet Baysal University local ethics committee (Date: 30/01/2019; Decision number: 2019/27).

\section{ORCID iD of the author(s)}

Mehmet Cosgunn / 0000-0002-6965-7444

Isa Sincer / 0000-0003-2399-9585

Yilmaz Gunes / 0000-0003-3817-851X

Zafer Kok / 0000-0001-7458-9135

Sule Aydin Turkoglu / 0000-0001-8616-832X
Copyrights: (C) 2021@author (s).

This is an open access article distributed under the terms of the Creative Commons Attribution License (CC BY 4.0), which permits unrestricted use, distribution, and reproduction in any medium, provided the original author(s) and source are credited and that the original publication in this journal is cited, in accordance with accepted academic practice. No use, distribution or reproduction is permitted which does not comply with these terms.

\section{References}

[1]Belbasis L, Bellou V, Evangelou E, et al. Environmental risk factors and multiple sclerosis: An umbrella review of systematic reviews and metaanalyses. Lancet Neurol. 2015;14(3):263-73.

[2]Sawcer S, Franklin RJ, Ban M. Multiple sclerosis genetics. Lancet Neurol. 2014;13(7):700-9.

[3]Brück, W, Stadelmann C. The spectrum of multiple sclerosis: new lessons from pathology. Curr Opin Neurol. 2005;18(3):221-24.

[4]Hohlfeld R, Kerschensteiner M, Stadelmann $\mathrm{C}$, et al. The neuroprotective effect of inflammation: implications for the therapy of multiple sclerosis. J Neuroimmunol. 2000;107(2):161-66.

[5]Sacks HS, Fain JN. Human epicardial adipose tissue: a review. Am Heart J. 2007;153(6): 907-17.

[6]Şengul C, Özveren O. Epicardial adipose tissue: a review of physiology, pathophysiology, and clinical applications. Anadolu Kardiyol Derg. 2013;13(3):261-65. [7]Mazurek T, Zhang L, Zalewski A, et al. Human epicardial adipose tissue is a source of inflammatory mediators. Circulation. 2003;108(20):2460-66. 
[8]Kim SH, Chung JH, Kwon BJ, et al. The Associations of Epicardial Adipose Tissue With Coronary Artery Disease and Coronary Atherosclerosis. Int Heart J. 2014;55(3):197-203.

[9]Imitola J, Chitnis T, Khoury SJ. Cytokines in multiple sclerosis: from bench to bedside. Pharmacol Ther. 2005;106(2):16377.

[10] Stys PK. Multiple sclerosis: autoimmune disease or autoimmune reaction? Can J Neurol Sci. 2010;37(S2):S16-S23.

[11]Asik M, Sahin S, Ozkul F, et al. Evaluation of epicardial fat tissue thickness in patients with Hashimoto thyroiditis. Clin Endocrinol. 2013;79(4):571-76.

[12]Fatma E, Bunyamin K, Savas S, et al. Epicardial fat thickness in patients with rheumatoid arthritis. Afr Health Sci. 2015;15(2):489-95.

[13] Iacobellis G, Barbaro G. The double role of epicardial adipose tissue as pro-and antiinflammatory organ. Horm Metab Res. 2008;40(07):442-45.

[14] Iacobellis G, Assael F, Ribaudo MC, et al. Epicardial fat from echocardiography: a new method for visceral adipose tissue prediction. Obes Res. 2003;11(2):304-10.

[15] Jadidi E, Mohammadi M, Moradi T. High risk of cardiovascular diseases after diagnosis of multiple sclerosis. Mult Scler. 2013;19(10):1336-40.

[16]Christiansen CF, Christensen S, Farkas $\mathrm{DK}$, et al. Risk of arterial cardiovascular diseases in patients with multiple sclerosis: a population-based cohort study. Neuroepidemiology. 2010;35(4):267 -74 .

[17] Allen NB, Lichtman JH, Cohen HW, et al. Vascular disease among hospitalized multiple sclerosis patients. Neuroepidemiology. 2008;30(4):2 34-38.

[18]Lindegard B. Diseases associated with multiple sclerosis and epilepsy. A population cohort study of 159,200 middleaged, urban, native Swedes observed over 10 years (1970-79). Acta Neurol Scand. 1985;71(4):267-77.

[19]Roshanisefat H, Bahmanyar S, Hillert J, et al. Multiple sclerosis clinical course and cardiovascular disease risk-S wedish cohort study. Eur J Neurol. 2014;21(11):1353-e88.

[20]Moccia M, Lanzillo R, Palladino R, et al. The Framingham cardiovascular risk score in multiple sclerosis. Eur J Neurol. 2015;22(8):1176-83.

[21] Yuksel B, Koc P, Ozaydin Goksu E, et al. Is multiple sclerosis a risk factor for atherosclerosis? J Neuroradiol. 2019; 7:S0150-9861(19)30462-66.

[22]Wens I, Dalgas U, Stenager E, et al. Risk factors related to cardiovascular diseases and the metabolic syndrome in multiple sclerosis - a systematic review. Mult Scler. 2013;19(12):1556-64.

[23]Pierdomenico SD, Pierdomenico AM, Neri $M$, et al. Epicardial adipose tissue and metabolic syndrome in hypertensive patients with normal body weight and waist circumference. Am J Hypertens. 2011;24(11):1245-49.

[24]Brück W, Stadelmann C. Inflammation and degeneration in multiple sclerosis. Neurol Sci. 2003;24(5):265-67.

[25]Frischer JM., Bramow S, Dal-Bianco A, et al. The relation between inflammation and neurodegeneration in multiple sclerosis brains. Brain. 2009;132(5):1175-89.

[26]Ramirez-Ramirez V, Macias-Islas MA, Ortiz GG, et al. Efficacy of fish oil on serum of TNF $\alpha$, IL- $1 \beta$, and IL- 6 oxidative stress markers in multiple sclerosis treated with 
interferon beta-1b. Oxid Med Cell Longev. 2013;2013:709493.

[27] Sharief MK, Hentges R. Association between tumor necrosis factor- $\alpha$ and disease progression in patients with multiple sclerosis. N Engl J Med. 1991;325(7): 46772 . 